\title{
Preparation and Screening of Protoplast Fusion Bacteria of Bacillus subtilis and Lactobacillus rhamnosus
}

\author{
Ye Ge ${ }^{1}$, Chunmiao Zhao ${ }^{1}$, Weitian $\mathrm{Xie}^{2}$, Ying $\mathrm{Liu}^{2, *}$ and Chunhou $\mathrm{Xu}^{1, *}$ \\ ${ }^{1}$ College of Coastal Agricultural Sciences, Guangdong Ocean University, Zhanjiang, \\ Guangdong Province, China \\ ${ }^{2}$ College of Food Science and Technology, Guangdong Ocean University, Zhanjiang, \\ Guangdong Province, China
}

\begin{abstract}
A B S T RA C T
Bacillus subtilis (B. subtilis) produces spores, and Rhamnose lactobacillus produces lactic acid. In this study, to obtain functional bacteria with lactic acid and lactobacilli and simultaneously producing spores and digestive enzymes, we produced the protoplast as the parent strain of B. subtilis and Rhamnose lactobacillus and analyzed the production rate of spores, the ability to resist high temperature and hereditary stability. The results showed that RH-3 was a perfect protoplast from which 48 strains were obtained through regeneration, with a sporogenic rate of $69.1 \%$ and a colony number of $2.7 \times 10^{5} \mathrm{CFU} / \mathrm{mL}$ after an $80^{\circ} \mathrm{C}$ high-temperature treatment. After 7 generations of fermentation, the average $\mathrm{pH}$ value was 4.47. The average number of colonies in the culture medium was 18.4. The production of protoplasts lays a foundation for research in microecological preparation.
\end{abstract}

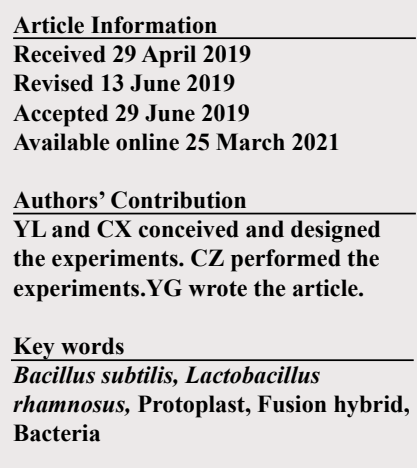

\section{INTRODUCTION}

$\mathrm{O}$ ne strategy for combining the desirable characteristics of a crop is interspecies hybridization by either sexual crosses or protoplast fusion. Protoplast fusion allows the transfer of organelle characteristics as well as agriculturally desirable nuclear traits between sexually incompatible species (Kang et al., 2017). Protoplast fusion, also known as cell fusion (Cheng et al., 2018), refers to the process by which the cells of the outer wall after protoplast formation and the cell wall by the release of enzymes through an external force (resultant or promotion by the melting agent) fuse and the protoplasts of two or more bacteria touch each other through membrane fusion (Hranueli et al., 1983), cytoplasmic fusion, and nuclear fusion, followed by contact and exchange between genomes, which occurs through genome genetic recombination; finally, the bacterial cell walls can undergo suitable regeneration and restructuring (Ge et al., 2014; Patil et al., 2015; Zhang et al., 2006b).

Microecological preparationisaliving microbiological preparation that can adjust the microecological balance of the body based on the theory of microecological balance,

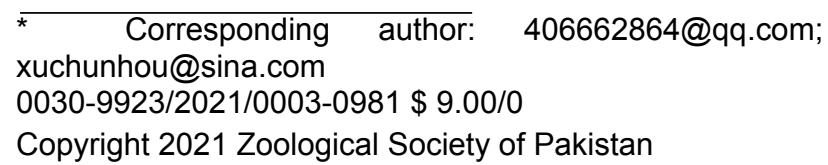

microecological imbalance, microecological nutrition and microecological prevention. Microecological preparation is also known as probiotics. As microecological preparations can supplement the quantity or type of normal microorganisms that are absent in the intestinal tract, they can adjust or maintain the intestinal microecological balance, enhance immune function, and promote the digestion and absorption of nutrients to achieve disease prevention, disease treatment, an increase in the feed conversion rate and an increase in the performance of livestock and poultry production. Bacillus subtilis produces spores and digestive enzymes and Lactobacillus rhamnosus produces lactic acid and lactobacilli, both of which are probiotics, but each has advantages and disadvantages. The purpose of this study was to utilize Bacillus subtilis and Lactobacillus rhamnosus as the parent strains and prepare protoplasts of these two strains and fuse them in order to obtain fusion strains (fusion bacteria) with advantageous characteristics of both parents. This study is expected to provide experimental data to lay the foundation for the development and application of Bacillus subtilis and Lactobacillus rhamnosus as microecological preparations in the future (Dai et al., 2005).

In this study, a fusion hybrid was obtained by the fusion of Bacillus subtilis and Lactobacillus rhamnosus. Regeneration and selection were tested using regeneration and screening methods.

MATERIALS AND METHODS 


\section{Bacterial strains}

Bacterial strains of Bacillus subtilis and Lactobacillus rhamnosus were supported by the microbiology lab of Guangdong Ocean University. Activated culture medium (Beef paste $5.0 \mathrm{~g}$, glucose $10.0 \mathrm{~g}$, peptone $10.0 \mathrm{~g}$, yeast paste $5.0 \mathrm{~g}, \mathrm{NaCl} 5.0 \mathrm{~g}$ in $1 \mathrm{~L}$ distilled water) of Bacillus subtilis (CM1), activated culture medium of Lactobacillus rhamnosus (CM2) (Beef paste $10.0 \mathrm{~g}$, glucose20.0 g, peptone10.0 g, yeast paste $5.0 \mathrm{~g}$, Twain $-801.0 \mathrm{~mL}$, $\mathrm{K} 2 \mathrm{PHO} 42.0 \mathrm{~g}$, sodium acetate $5.0 \mathrm{~g}$, triammonium citrate $2.0 \mathrm{~g}, \mathrm{MgSO} 4.7 \mathrm{H} 2 \mathrm{O} 0.2 \mathrm{~g}, \mathrm{MnSO} 40.05 \mathrm{~g}, 1 \mathrm{~L}$ distilled water) and regeneration medium were prepared by our lab.

Growth curves of Bacillus subtilis and Lactobacillus rhamnosus

Bacterial strains were inoculated in activated medium and cultured at $37{ }^{\circ} \mathrm{C}$. Culture samples were taken every $2 \mathrm{~h}$ and tested using an absorbance wavelength of $600 \mathrm{~nm}$ (De Leersnyder et al., 2018; Zhang et al., 2016).

\section{The fusion of protoplasts}

The protoplast suspension was acquired according to standard methods. One clone each of Bacillus subtilis and Lactobacillus rhamnosus was picked from the slant medium and placed into $50 \mathrm{ml} \mathrm{CM} 1$ and CM2 media for activation for $14 \mathrm{~h}$ at $37^{\circ} \mathrm{C}$. The strains were further cultured with $2 \%$ inoculation and at $36^{\circ} \mathrm{C}$ oscillation until the culture reached logarithmic growth, and penicillin and glycine were added. The culture continued shaking until reaching late logarithmic growth.

The cells were centrifuged at $4000 \mathrm{r} / \mathrm{min}$ for $15 \mathrm{~min}$, and the supernatant was discarded. The cells were washed twice with PBS. The lysozyme was used for Bacillus subtilis $(2 \mathrm{mg} / \mathrm{ml})$ and Lactobacillus rhamnosus $(4 \mathrm{mg} / \mathrm{ml})$. After enzymatic hydrolysis, the protoplast was precipitated after $4000 \mathrm{r} / \mathrm{min}$ centrifugation for $15 \mathrm{~min}$. The protoplast was washed twice with PBS and then suspended in $5 \mathrm{ml}$ hypertonic buffer (SMM).

The protoplasts of Bacillus subtilis and Lactobacillus rhamnosus were mixed with the same volumes, and after a static $5 \mathrm{~min}$, the mixture was centrifuged for $15 \mathrm{~min}$ at 4000 $\mathrm{r} / \mathrm{min}$, and the supernatant was discarded. Polyethylene glycol (PEG) and new calcium phosphate solution were added into the protoplast mixture, incubated at $37{ }^{\circ} \mathrm{C}$ for $20 \mathrm{~min}$, and then centrifuged at $4000 \mathrm{r} / \mathrm{min}$ for $15 \mathrm{~min}$. The obtained protoplast fusion was put into a high suspended solution and preserved at $4{ }^{\circ} \mathrm{C}$.

\section{Regeneration and screening of the fusion hybrid}

The existence of the spores was screened with a microscope, and spores were put into a $0.1 \mathrm{ml}$ fusion suspension of different $\mathrm{pH}$ values on a solid medium plate and incubated at $36{ }^{\circ} \mathrm{C}$ for $24 \sim 48 \mathrm{~h}$, and the rate of spore regeneration, the ability to resist high temperature, the stability of acid production and the genetic stability were determined according to conventional methods (Alander et al., 1999).

\section{RESULTS}

\section{Growth curves}

The growth curves of Bacillus subtilis and Lactobacillus rhamnosus are shown in Figure 1. The growth curves showed that Bacillus subtilis grew slowly after $4 \mathrm{~h}$ inoculation, and then the growth rate increased sharply, reaching a peak at $6 \mathrm{~h}$ and decreasing after $8 \mathrm{~h}$ to the end stage of logarithmic growth. The growth rate of Lactobacillus rhamnosus increased slowly before $10 \mathrm{~h}$; however, the growth rate increased sharply and reached its peak at $12 \mathrm{~h}$. The end stage of logarithmic growth occurred at $14 \mathrm{~h}$.
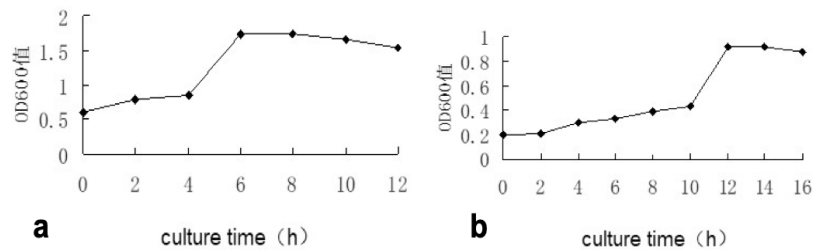

Fig. 1. Growth curves of Bacillus subtilis and Lactobacillus rhamnosus. The incubation time was used as the abscissa, and the absorbance was used as ordinate to plot on the plot. The mid-logarithmic growth phase of these two strains was determined.

\section{Protoplast fusion}

The protoplast is the spheroid after removing the cell wall, which is round or oval with a visible nucleus. Under the action of external forces, the protoplast of Bacillus subtilis (Fig. 2a) and Lactobacillus rhamnosus (Fig. $2 b$ ) interacted with each other and formed a new fusion bacterium through membrane fusion, cytoplasmic fusion and nuclear fusion (Fig. 2c).

\section{Protoplast regeneration}

Nine strains were produced as determined by smear and staining microscopy, and the $\mathrm{pH}$ values from 48 regenerated strains, which were named $\mathrm{RH}-1, \mathrm{RH}-2, \mathrm{RH}-$ 3, RH-4, RH-5, RH-6, RH-7, RH-8 and RH-9 (Table I), were determined. The $\mathrm{pH}$ of the parent strains Bacillus subtilis and Lactobacillus rhamnosus under the same conditions were 7.15 and 3.44, respectively. The $\mathrm{pH}$ values of the 9 regenerated strains were all between the $\mathrm{pH}$ values 
of the two parent strains, of which the lowest $\mathrm{pH}$ value was $\mathrm{RH}-3$, at 4.32 .

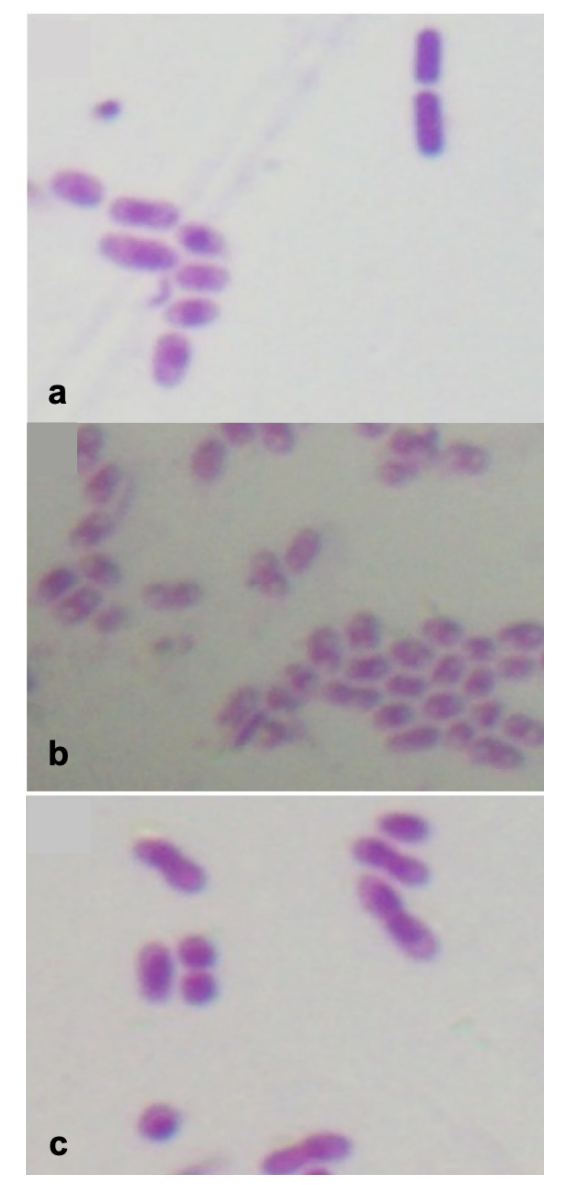

Fig. 2. Fusion of the protoplast of the two strains; a, Protoplast of Bacillus subtilis; b, Protoplast of Lactobacillus rhamnosus; c, Fusion of the protoplast.

Table I.- pH value of generated bacteria strains.

\begin{tabular}{ll}
\hline Strains & $\mathbf{p H}$ \\
\hline RH-1 & 4.75 \\
RH-2 & 4.69 \\
RH-3 & 4.32 \\
RH-4 & 4.67 \\
RH-5 & 4.73 \\
RH-6 & 4.43 \\
RH-7 & 4.62 \\
RH-8 & 4.76 \\
RH-9 & 4.47 \\
\hline
\end{tabular}

Screening of the protoplasts

The spore-generation rate of the 9 strains, both sporeproducing and acid-producing strains, were analyzed, and the results are shown in Table II. The spore yield of Bacillus subtilis was $71.5 \%$. The spore yields of the 9 strains were all lower than those of Bacillus subtilis. The strain with the highest spore yield was RH-9, at 69.1\%. The fusion protoplasts, with production rates of spores lower than $50 \%$, were discarded.

Table II.- Sporulation efficiency of generated bacteria strains.

\begin{tabular}{ll}
\hline Bacteria strains & Sporulation efficiency \% \\
\hline RH-1 & 25.5 \\
RH-2 & 56.7 \\
RH-3 & 69.1 \\
RH-4 & 39.8 \\
RH-5 & 56.3 \\
RH-6 & 61.2 \\
RH-7 & 57.0 \\
RH-8 & 63.2 \\
RH-9 & 59.6 \\
\hline
\end{tabular}

Bacillus subtilis strains had a strong ability to resist high temperature; there was $8.5 \times 10^{6} \mathrm{CFU} / \mathrm{ml}$ in the medium after an $80{ }^{\circ} \mathrm{C}$ heat treatment for $15 \mathrm{~min}$. The strains of Lactobacillus rhamnosus, RH-6 and RH-7, had a poorer ability to resist high temperature. There were no colonies in the medium after the $80{ }^{\circ} \mathrm{C}$ heat treatment for $15 \mathrm{~min}$. The number of bacterial colonies of RH-3, RH-5 and RH-9 were less than those of Bacillus subtilis, but the difference was not obvious. However, the number of bacterial colonies grown on the culture medium after hightemperature treatment of strains $\mathrm{RH}-2$ and $\mathrm{RH}-8$ was fewer, $3.8 \times 10^{2} \mathrm{CFU} / \mathrm{ml}$ and $2.5 \times 10^{2} \mathrm{CFU} / \mathrm{ml}$, respectively, and they were still able to withstand a certain high temperature. Five strains, RH-2, RH-3, RH-5, RH-8 and RH-9, were preserved in this test for further study (Table III).

The study of the stability of acid production showed that the acid production curves of strains RH-2 and RH-8 fluctuated greatly (Fig. 3), indicating that the acid production energy was unstable. However, the acid production curves of strains RH-3, RH-5 and RH-9 had little fluctuation and stable acidity, and their average $\mathrm{pH}$ values were 4.47, 4.71 and 4.49 respectively. Based on the results of the stability of acid production of the 5 regenerated strains, the strains with a stable acid production capacity, RH-3, RH-5 and RH-9, were preserved for further study (Fig. 3). 
Table III.- Temperature capabilities of 7 regenerated bacteria strains.

\begin{tabular}{llll}
\hline Bacteria strains & \multicolumn{3}{c}{ Number/ (CFU/mL) } \\
\hline Temperature & $56^{\circ} \mathrm{C}$ & $65^{\circ} \mathrm{C}$ & $80^{\circ} \mathrm{C}$ \\
RH-2 & $5.610^{6}$ & $2.4 \times 10^{4}$ & $3.8 \times 10^{2}$ \\
RH-3 & $5.4 \times 10^{6}$ & $2.1 \times 10^{6}$ & $2.7 \times 10^{5}$ \\
KCRH-5 & $6.1 \times 10^{6}$ & $1.9 \times 10^{6}$ & $2.3 \times 10^{3}$ \\
RH-6 & $4.3 \times 10^{6}$ & $5.9 \times 10^{4}$ & 0 \\
RH-7 & $5.6 \times 10^{5}$ & $1.9 \times 10^{3}$ & 0 \\
RH-8 & $6.2 \times 10^{5}$ & $4.2 \times 10^{3}$ & $2.5 \times 10^{2}$ \\
RH-9 & $7.8 \times 10^{6}$ & $3.2 \times 10^{6}$ & $1.2 \times 10^{5}$ \\
KC & $8.5 \times 10^{6}$ & $6.0 \times 10^{6}$ & $5.9 \times 10^{5}$ \\
LGG & $4.6 \times 10^{5}$ & $1.2 \times 10^{3}$ & 0 \\
\hline
\end{tabular}

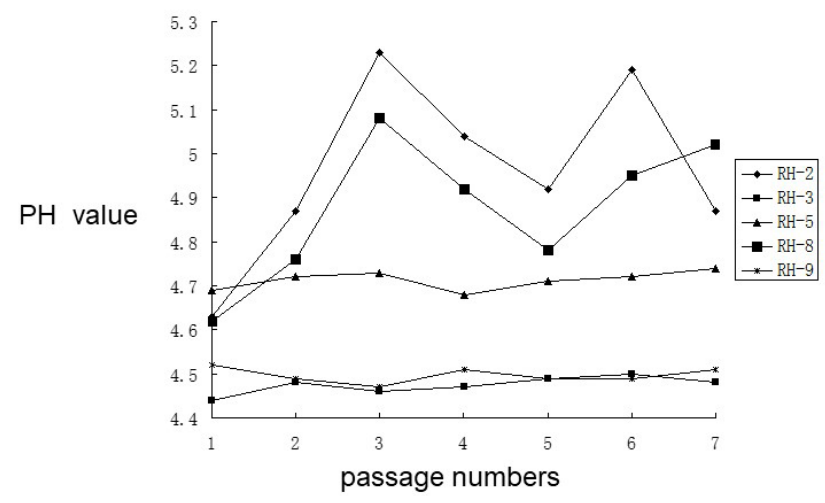

Fig. 3. Acid production stability of the 5 regenerated bacterial strains.

The genetic stability of the 3 regenerated strains, RH-3, RH-5 and RH-9, were analyzed (Fig. 4). The results showed that the colony number curve of colony RH-5 fluctuated greatly. Although the growth was stable in the late stage, the number of colonies was low. After 7 generations of protoplast culture, the average number of colonies was 14.3 strains. The colonies of RH-3 and RH-9 were relatively flat and were genetically stable. The average number of the two colonies were 18.4 and 19.1 after seven generations.

The bacterial strain RH-3 was retained as a relatively ideal fusion strain after comprehensive evaluation of the $\mathrm{pH}$ and the spore production rate.

The colony morphology and microscopic characteristics were analyzed for the RH-3 fusion strain and the parental strains of Bacillus subtilis and Lactobacillus rhamnosus in solid medium. The bacterial colonies of Bacillus subtilis were of medium size, and the diameter was 2 5 $\mathrm{mm}$. The bacterial surface had umbrella-shaped folds, which were rough and opaque in color. Microscopy was utilized to characterize Gram staining positivity, long rod shape, and sporulation (Fig. 5a). The colonies of Lactobacillus rhamnosus were small, approximately $1 \sim 2 \mathrm{~mm}$ in diameter, with a smooth surface and an opaque milky white color. Microscopy was utilized to characterize Gram staining positivity, a short rod shape, and no sporulation (Fig. 5b). The colony size of the RH-3 fusion strain was between the two parent strains, with a diameter of approximately $2 \sim 3 \mathrm{~mm}$. The surface was flat, white and opaque. Microscopy was utilized to characterize Gram staining positivity, rod shape, and bacterial size between the two parental strains, as well as sporulation (Fig. 5c).

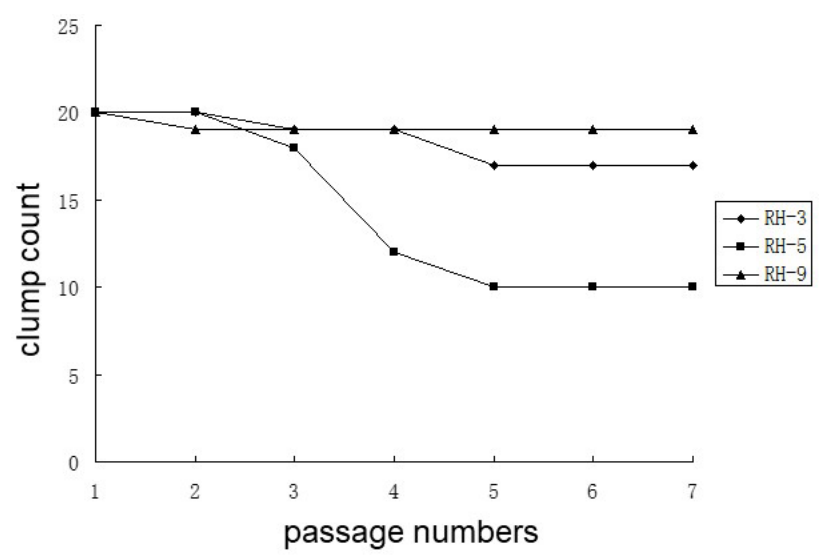

Fig. 4. Genetic stability of the 3 regenerated bacterial strains.
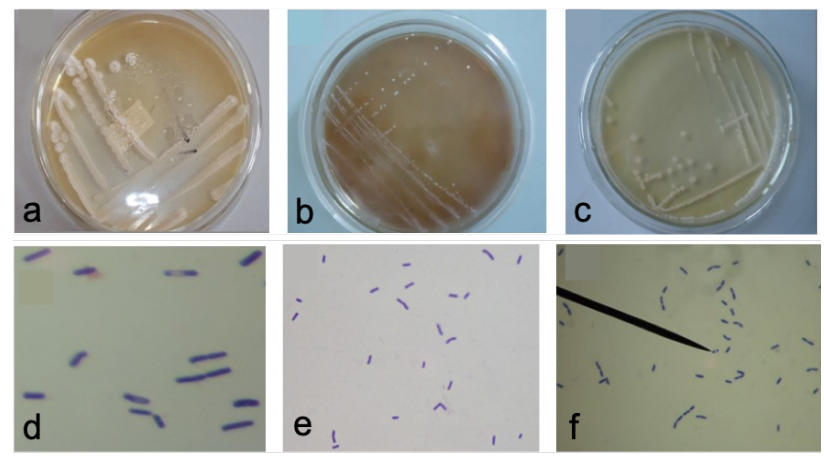

Fig. 5. An image of growth on solid medium of three bacterial strains and a microscopy image of three bacterial strains $(10 \times 100)$ : a and d were Bacillus subtilis, $\mathrm{b}$ and $\mathrm{e}$ were Lactobacillus rhamnosus and $\mathrm{c}$ and $\mathrm{f}$ were fusion of RH-3. 


\section{DISCUSSION}

The growth and reproduction of bacteria are fluctuating processes that are divided into four periods: a sluggish period, logarithmic period, stable period and declining period; each period has its own characteristics. The physiological state of bacteria is an important factor that can affect the preparation of protoplasm. If the bacteria are too old, the cytoderm ages and thickens; however, if the bacteria are too young, the cytoderm breaks easily. Having too old and/or young of bacteria make protoplasts hard to obtain. Most studies have shown that the growth and metabolism of bacteria in the logarithmic growth stage were vigorous, and the cytoderm was most sensitive to lysozymes. Currently, the prepared protoplast formation rate and regeneration rate are higher. In this study, the OD values of Bacillus subtilis and Lactobacillus rhamnosus were measured every two hours under a spectrophotometer with a wavelength of $600 \mathrm{~nm}$, the growth curves of the two bacteria were obtained, and their logarithmic growth periods were determined to be $6 \mathrm{~h}$ and $12 \mathrm{~h}$, respectively.

Glycine and penicillin can increase the sensitivity of the cytoderm to lysozyme within a certain concentration range (Zhang et al., 2006). The enzymatic hydrolysis method is commonly used to remove the bacterial cytoderm. Because the composition and structure of the cytoderm of different strains are different, the types of enzymes used by different strains are different. Reportedly, lysozymes can be used to prepare the protoplast of grampositive bacteria. In this study, we added lysozymes to the enzymolysis experiment.

There are many reports on the fusion methods of protoplasts, among which Ferenczy in Hungary reported the fusion of a protoplast by using the centrifugal force induction method for nutritional-deficient mutant strains of mildew in 1974 (Kano et al., 2004). In this study, chemical fusion was used. If only the protoplasts of Bacillus subtilis and Lactobacillus were mixed together, the fusion rate was low, PEG and $\mathrm{Ca} 2+$ needed to be added to improve the fusion rate.

The colony characteristics formed by the growth of different microorganisms in culture medium are substantially different, while the colony characteristics of the same bacteria under certain conditions have a certain level of stability, which can be used to distinguish different bacteria. Therefore, the culture characteristics of microorganisms are also important content in microbial screening and identification. In this study, 9 target strains were selected for further screening by morphological observation and the study of spore-producing and acidic energy generation.

Strains were preliminarily targeted and evaluated further. This research adopted the spore production rate, acid yield stability, high temperature resistant ability and genetic stability identification methods to obtain an ideal fusion strain, namely, the RH-3 fusion strain, whose cultures had a $\mathrm{pH}$ value of 4.32 and a spore production rate of $69.1 \%$. This $\mathrm{pH}$ value was larger than that of Bacillus subtilis (7.15) and then decreased by $39.6 \%$, and there was very little difference in the rate of spore production of Bacillus subtilis of $71.5 \%$ (71.5\%).

In conclusion, we acquired one protoplast that had lactic acid and lactobacilli and simultaneously produced spores and digestive enzymes. The fusion protoplast may provide the basis for the research and development of microecological preparations.

\section{ACKNOWLEDGEMENTS}

The author thanks Weitian Xie for his assistance in research advice.

Statement of conflicts of interest

All the authors have no conflicts of interest.

\section{REFERENCES}

Alander, M., Satokari, R., Korpela, R., Saxelin, M., Vilpponen-Salmela, T., Mattila-Sandholm, T. and von Wright, A., 1999. Persistence of colonization of human colonic mucosa by a probiotic strain, Lactobacillus rhamnosus GG, after oral consumption. Appl. environ. Microbiol., 65: 351354.

Cheng, T., Xu, X., Zhang, W., Chen, L. and Liu, T., 2018. Protoplast preparation from enriched flagellates and resting cells of Haematococcus pluvialis. J. appl. Microbiol., 124: 469-479. https:// doi.org/10.1111/jam.13643

Dai, M., Ziesman, S., Ratcliffe, T., Gill, R.T. and Copley, S.D., 2005. Visualization of protoplast fusion and quantitation of recombination in fused protoplasts of auxotrophic strains of Escherichia coli. Metab. Eng., 7: 45-52. https://doi.org/10.1016/j. ymben.2004.09.002

De Leersnyder, I., De Gelder, L., Van Driessche, I. and Vermeir, P., 2018. Influence of growth media components on the antibacterial effect of silver ions on Bacillus subtilis in a liquid growth medium. Sci. Rep., 8: 9325. https://doi.org/10.1038/s41598-01827540-9

Ge, J.P., Zhao, J.W., Zhang, L.Y., Zhang, M.Y. and Ping, W.X., 2014. Construction and analysis of highethanol-producing fusants with co-fermentation 
ability through protoplast fusion and double labeling technology. PLoS One, 9. https://doi. org/10.1371/journal.pone.0108311

Hranueli, D., Pigac, J., Smokvina, T. and Alacevic, M.,1983. Genetic interactions in Streptomyces rimosus mediated by conjugation and by protoplast fusion. J. Gen. Microbiol., 129: 1415-1422. https:// doi.org/10.1099/00221287-129-5-1415

Kang, L., Li, P.F., Wang, A.F., Ge, X.H. and Li, Z.Y., 2017. A novel cytoplasmic male sterility in Brassica napus (inap CMS) with carpelloid stamens via protoplast fusion with Chinese woad. Front. Plant. Sci., 8: 529. https://doi.org/10.3389/ fpls.2017.00529

Kano, S., Aimi, T., Masumoto, S., Kitamoto, Y. and Morinaga, T., 2004. Physiology and molecular characteristics of a pine wilt nematode-trapping fungus, Monacrosporium megalosporum. Curr. Microbiol., 49: 158-164. https://doi.org/10.1007/ s00284-004-4268-9

Patil, N.S., Patil, S.M., Govindwar, S.P. and Jadhav, J.P., 2015. Molecular characterization of intergeneric hybrid between Aspergillus oryzae and Trichoderma harzianum by protoplast fusion. J. appl. Microbiol., 118: 390-398. https://doi.org/10.1111/jam.12711

Zhang, H.Q., Liu, Y.Q., Liu, B. and Gao, P.J., 2006. A novel approach for estimating growth phases and parameters of bacterial population in batch culture. Sci. China Ser. C., 49: 130-140. https://doi. org/10.1007/s11427-006-0130-6

Zhang, K.D., Zhang, B.Y., Chen, B., Jing, L., Zhu, Z.W. and Kazemi, K., 2016. Modeling and optimization of Newfoundland shrimp waste hydrolysis for microbial growth using response surface methodology and artificial neural networks. Mar. Pollut. Bull., 109: 245-252. https://doi. org/10.1016/j.marpolbul.2016.05.075

Zhang, Y., Lin, S.M., Zhu, Y.J., Liu, C.J., Dong, Y., Li, F.F., Wu, G.F., Wang, H.Y. and Zhang, J.H., 2006 b. Protoplast fusion between Geotrichum candidium and Phanerochaete chrysosporium to produce fusants for corn stover fermentation. Biotechnol. Lett., 28: 1351-1359. https://doi.org/10.1007/ s10529-006-9097-4 